\title{
Ground-state optimization and hysteretic demagnetization: The random-field Ising model
}

\author{
Mikko J. Alava, ${ }^{1}$ Vittorio Basso, ${ }^{2}$ Francesca Colaiori, ${ }^{3}$ Lorenzo Dante, ${ }^{3}$ Gianfranco Durin,${ }^{2}$ \\ Alessandro Magni, ${ }^{2}$ and Stefano Zapperi ${ }^{3}$ \\ ${ }^{1}$ Helsinki University of Technology, Laboratory of Physics, HUT-02105 Espoo, Finland \\ ${ }^{2}$ Istituto Elettrotecnico Nazionale Galileo Ferraris, strada delle Cacce 91, 10135 Torino, Italy \\ ${ }^{3}$ INFM unità di Roma 1 and SMC, Dipartimento di Fisica, Università “La Sapienza," Piazzale Aldo Moro 2, 00185 Roma, Italy
}

(Received 12 July 2004; revised manuscript received 29 November 2004; published 28 February 2005)

\begin{abstract}
We compare the ground state of the random-field Ising model with Gaussian distributed random fields, with its nonequilibrium hysteretic counterpart, the demagnetized state. This is a low-energy state obtained by a sequence of slow magnetic-field oscillations with decreasing amplitude. The main concern is how optimized the demagnetized state is with respect to the best-possible ground state. Exact results for the energy in $d=1$ show that in a paramagnet, with finite spin-spin correlations, there is a significant difference in the energies if the disorder is not so strong that the states are trivially almost alike. We use numerical simulations to better characterize the difference between the ground state and the demagnetized state. For $d \geqslant 3$, the random-field Ising model displays a disorder induced phase transition between a paramagnetic and a ferromagnetic state. The locations of the critical points $R_{c}^{(\mathrm{DS})}$ and $R_{c}^{(\mathrm{GS})}$ differ for the demagnetized state and ground state. We argue based on the numerics that in $d=3$ the scaling at the transition is the same in both states. This claim is corroborated by the exact solution of the model on the Bethe lattice, where the critical points are also different.
\end{abstract}

DOI: 10.1103/PhysRevB.71.064423

PACS number(s): 75.60.Ej, 02.60.Pn, 75.50.Lk

\section{INTRODUCTION}

The relation between equilibrium and nonequilibrium states is a central problem in the physics of disordered systems. Disorder induces a multitude of metastable states in which the system can easily be trapped. The dynamics is usually very slow, or glassy, and on observational time scales the system is basically always out of equilibrium. On the other hand, from the theoretical point of view it is easier to consider equilibrium properties, since in this case it is possible to use all the machinery of statistical physics to tackle the problem. The question is whether the equilibrium properties of disordered systems provide a faithful representation of the nonequilibrium states in which the system is likely to be found in practice. This dichotomy is at the core of many unsolved issues in the field of disordered system. Typical quantities that one could compare are the energy, the geometric characterization of the state (as domains in magnets), and the energy cost of excitations.

A simplification of the problem is obtained considering only athermal processes, in which the temperature of the system plays no role and can be ignored. The equilibrium state is in this case just the ground state (GS), the state of minimal energy. ${ }^{1}$ A zero temperature, nonequilibrium dynamics is purely relaxational: the system falls simply in the closest metastable state. A convenient way to allow the system to explore the various metastable states is by applying an external magnetic field. Different field histories typically result in hysteresis and lead to different metastable configurations. ${ }^{2}$

The demagnetization process consists in applying a slowly varying ac field with decreasing amplitude, and provides a simple way to access low-energy states. ${ }^{2}$ It has been studied for more than a century, but until recently the question how close the demagnetized state (DS) is to the GS was not addressed. This is the concern of our work, the problem of how such an optimization process works in the case of a random magnet. Recently, Zarand et al. have proposed the demagnetization process as the basis for a new optimization algorithm for disordered systems. ${ }^{3}$ The idea behind such "hysteretic optimization," is that demagnetization leads to a low-energy state, sufficiently close to the GS, which can then be reached by applying other methods using the DS as an input. The method was tested for different models such as spin glasses and NP-hard problems.

Here, we will concentrate on the random-field Ising model (RFIM), which, while retaining some complex features characteristic of disordered systems, still allows for a theoretical analysis. ${ }^{4}$ In the RFIM, due to the absence of frustration, the equilibrium state is relatively simple, however, the nonequilibrium dynamics is far from trivial. Due to the coupling of the local disorder to the order parameter, even the GS presents a variety of phenomena, which can be studied numerically. ${ }^{5-8}$ In fact the GS is found in a polynomial CPU time, with exact combinatorial algorithms, ${ }^{1}$ and solved exactly in $d=1$ and on the Bethe lattice. ${ }^{9,10}$ The equilibrium critical exponents for random-field magnets have been measured experimentally in $\mathrm{Fe}_{0.93} \mathrm{Zn}_{0.07} \mathrm{~F}_{2}$ antiferromagnets. ${ }^{11,12}$

The hysteretic properties of nonequilibrium RFIM have been widely studied in the recent literature. The hysteresis loops display a disorder induced phase transition: for low disorder the loop has a macroscopic jump at the coercive field, while at high disorder the loop is smooth, at least on the macroscopic scale. ${ }^{13-15}$ At smaller scale the magnetization curve is highly discontinuous, showing Barkhausen-type bursts, in correspondence to jumps between different metastable states. ${ }^{16} \mathrm{~A}$ disorder induced nonequilibrium phase transition in the hysteresis loop has been studied experimentally in Co-CoO films ${ }^{17}$ and Cu-Al-Mn alloys. ${ }^{18}$ 


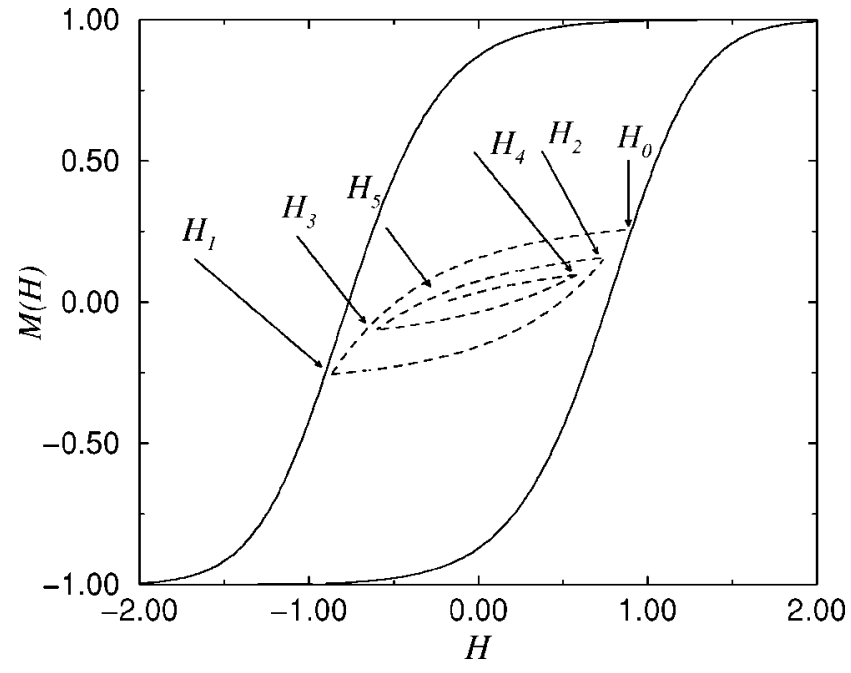

FIG. 1. The hysteresis loop of the RFIM computed exactly in $d=1$. We also report three minor loops. A demagnetization procedure corresponds to a series of minor loops obtained reversing the field at $H_{0}, H_{1}, H_{2}, \ldots$ as detailed in the text.

Extensive numerical simulations have been used to characterize disorder induced transitions in the nonequilibrium RFIM and critical exponents have been estimated in several dimensions. ${ }^{15,19,20}$ The model has been studied by the renormalization group and the exponents have been computed in a $\epsilon=6-d$ expansion. ${ }^{14}$ In addition the hysteresis loop has been computed exactly in $d=1$ and on the Bethe lattice, where the disorder induced transition is present for sufficiently large coordination number. While in $d=1$ there is definitely no transition, the situation in $d=2$ is less clear. Recently the problem of minor loops has been tackled analytically and numerically. Minor loops are obtained by reversing the field before the magnetization has reached saturation (see Fig. 1). In particular, the demagnetization curve has been computed exactly in $d=1$ (Ref. 21) and on the Bethe lattice, ${ }^{22}$ extending previous calculations ${ }^{23-27}$ of minor loops.

The equilibrium properties of the RFIM are governed by a zero-temperature fixed point, and in finite dimensions $(d$ $<5$ in practice) GS calculations have elucidated the properties of the phase diagram. In $d \geqslant 3$, the GS displays a ferromagnetic phase transition induced by the disorder ${ }^{1,8}$ (for $d$ $=4$, see Refs. 28,29). As domain-wall energy arguments and exact mathematical results indicate, in $d=2$ there is no phase transition but an effective ferromagnetic regime for small systems, while in $d=1$ the RFIM is trivially paramagnetic. It has been suggested that the transition in the GS is ruling the transition in the nonequilibrium hysteresis loop, also because mean-field calculations give the same results in and out of equilibrium. ${ }^{30}$ Numerical values of the exponents are close but not equal, but one must consider the difficulties in extrapolating values from the finite-size scaling. ${ }^{30,31}$ More recently, the question of the universality of the exponents, with respect to the shape of the disorder distribution, was discussed in $d=3$ simulations, mean-field theory, and on the Bethe lattice. $^{32-34}$

Below we report a detailed comparison of the zerotemperature equilibrium and nonequilibrium properties of the RFIM with Gaussian distribution of the random fields. We first analyze the problem in $d=1$, where exact results can be obtained. The average value of the energy is computed as a function of the disorder strength for the DS and the GS. A direct comparison of the two values shows that for weak disorder the differences become more substantial, while for strong disorder, where each spin basically aligns with the random field, the difference tends to vanish. Numerical studies using the same disorder realizations reveal that the main difference between the two states comes from the complete reversal of GS domains in the DS. This is also visible in the overlap between the GS and DS.

We then study the $d=3$ case in which both paramagnetic and ferromagnetic behavior exist. The question of whether the transitions appearing in the GS and in the hysteresis loop are universal has often been debated in the literature. ${ }^{30,31} \mathrm{At}$ the mean-field level it is not possible to distinguish the equilibrium from the nonequilibrium case and the transition is thus trivially the same. In addition, the $\epsilon$ expansion for the equilibrium and hysteretic transitions is the same to all orders, but one should always consider the possibility of nonperturbative corrections to the field theory. Numerical simulations in $d=3$ indicate that the critical exponents and the critical disorder in the two transitions are reasonably close, but the numerical uncertainties do not allow for a conclusive statement about their identity. Here we directly compare the behavior of the GS and the DS in $d=3$ close to the disorder induced phase transitions. We show that while the nonuniversal critical parameter $R_{c}$ differs in the two cases, the universal finite-size scaling curve for the order parameter can be collapsed on the same curve. This suggests some kind of universality in the GS and the DS transitions. The numerical simulations for the GS and DS are done for the same disorder realizations for both the cases, for cubic lattices of linear sizes $L=10,20,40,80$. The results are averaged over several realizations of the quenched random fields. In both cases, we compute the average magnetization as a function of the disorder width.

A difference in the location of the critical point for equilibrium and nonequilibrium behavior of the same model may appear rather peculiar and one could be tempted to ascribe it to finite-size corrections. In order to clarify this issue, we have solved exactly the model on the Bethe lattice and compared the results for GS and DS. While the exponents, as expected, are the same, coinciding with the results of meanfield theory, the critical disorder differs in the two cases. Namely, the transition in the DS occurs at a lower disorder value. Thus there is an intermediate parameter region where the GS is ferromagnetic but the DS is paramagnetic. The solution on the Bethe lattice corroborates the picture obtained from simulations in $d=3$. From the optimization viewpoint, the $d=3$ case shows an intermediate phase of "bad" correspondence between the GS and DS, exactly as in $d=1$. This, however, stops as $R_{c}^{(\mathrm{DS})}$ is approached: naturally if both the states are ferromagnetic the optimization of the DS is much easier. To further explore the question of universality of the two transitions in the GS and in the DS, we have computed the distribution of the magnetization at the respective critical point, $R_{c}^{(\mathrm{DS})}$ and $R_{c}^{(\mathrm{GS})}$, for different lattice sizes. The distributions can again all be collapsed into the same curve. 
Finally, we consider the question of when is it actually possible to reach the GS via a generic field history. To this end, we consider a proper algorithm that allows in principle to construct a field history, provided that it exists, which brings to the GS. Studies of the $d=1$ case illuminate the difficulty of optimizing since it turns out that for anything but very strong disorders, $R$ the probability to reach the GS rapidly decays to zero.

Our main conclusion is that, in general, demagnetization is not a good technique for reaching states that are truly close to the equilibrium, except in cases where the outcome is clearly similar from the very beginning (ferromagnetic states and paramagnetic states where the disorder is strong). This holds for both the energy of the states and also for the spin configurations. A simple formulation is that, since in the DS the locations of domain walls are not optimized, there is an excess random-field (Zeeman) energy.

The paper is organized as follows: in Sec. II we define the model and discuss its numerical treatment. In Sec. III we analyze the one-dimensional case, analytically and numerically. Section IV is devoted to the behavior around the disorder induced transition in $d=3$ and on the Bethe lattice. Section V demonstrates the algorithm to reconstruct the field history, together with numerical studies. Conclusions are reported in Sec. VI. An account of some of these results was briefly reported in Ref. 35 .

\section{THE RANDOM-FIELD ISING MODEL}

In the RFIM, a spin $s_{i}= \pm 1$ is assigned to each site $i$ of a $d$-dimensional lattice. The spins are coupled to their nearestneighbor spins by a ferromagnetic interaction of strength $J$ and to the external field $H$. In addition, to each site of the lattice is associated a random field $h_{i}$ taken from a Gaussian probability $\rho(h)=\exp \left(-h^{2} / 2 R^{2}\right) / \sqrt{2 \pi} R$, with standard deviation $R$. The Hamiltonian thus reads

$$
\mathcal{H}=-\sum_{\langle i, j\rangle} J_{s_{i}} s_{j}-\sum_{i}\left(H+h_{i}\right) s_{i},
$$

where the first sum is restricted to nearest-neighbor pairs.

In this paper we will consider only the case of zero temperature, both in equilibrium and out of equilibrium. The $T$ $=0$ equilibrium problem amounts to find the minimum of $\mathcal{H}$ for a given realization of the random fields (i.e., the GS) and then eventually perform the thermodynamic limit. This problem has been solved exactly in a number of simple cases, namely, in $d=1$ and on the Bethe lattice, for particular disorder distributions and studied numerically in generic dimensions.

The RFIM GS is solvable in a polynomial CPU time, with exact combinatorial algorithms. For the one-dimensional case, the solution can be found via a mapping to a "shortest path problem" ${ }^{36}$ which effectively places the domain walls in optimal positions, corresponding to the global minimum of $\mathcal{H}$. For higher dimensions, one starts by noticing that finding the RFIM GS is equivalent to the min-cut/max-flow problem of combinatorial optimization. This can be solved in a variety of ways. We use a so-called push-relabel variant of the preflow algorithm. ${ }^{37}$ Such methods, properly implemented, perform in general only slightly worse than linear as a function of the number of spins in the problem.

For the out of equilibrium case, we need to specify an appropriate dynamics, ruling the evolution of the spins. We will consider the dynamics proposed in Ref. 38 and used in Refs. 13-15 to study the hysteresis loop. At each time step the spins align with the local field

$$
s_{i}=\operatorname{sign}\left(J \sum_{j} s_{j}+h_{i}+H\right),
$$

until a metastable state is reached. This dynamics can be used to obtain the hysteresis loop. The system is started from a state with all the spin down $s_{i}=-1$ and then $H$ is ramped slowly from $H \rightarrow-\infty$ to $H \rightarrow \infty$. The limit of $d H / d t \rightarrow 0$ can be conveniently obtained by increasing the field precisely of the amount necessary to flip the first unstable spin. A single spin flip increases the local field of the nearest-neighboring spins, generating an avalanche of flippings. When the systems find another metastable state, the field is increased again. This dynamics obeys return-point memory: $:^{13}$ if the field is increased adiabatically the magnetization only depends on the state in which the field was last reversed. This property has been exploited in $d=1$ (Refs. 21,24) and in the Bethe lattice 22,27 to obtain exactly the saturation cycle and the minor loops.

The main hysteresis loop selects a series of metastable states, which in principle are not particularly close to the ground state. To obtain low-energy states, we perform a demagnetization procedure: the external field is changed through a nested succession $H=H_{0} \rightarrow H_{1} \rightarrow H_{2} \rightarrow \cdots \rightarrow H_{n}$ $\rightarrow \cdots \rightarrow 0$, with $H_{2 n}>H_{2 n+2}>0, H_{2 n-1}<H_{2 n+1}<0$, and $d H$ $\equiv H_{2 n}-H_{2 n+2} \rightarrow 0$. A perfect demagnetization can be performed numerically using the prescription discussed above to obtain $d H / d t \rightarrow 0$. Such a perfect demagnetization is quite expensive computationally and it is convenient to perform an approximate demagnetization using $d H=10^{-3}$. A comparison of the states obtained under approximate and perfect demagnetization shows negligible differences.

\section{GROUND STATE AND DEMAGNETIZED STATE IN ONE DIMENSION}

\section{A. Exact results: Ground State}

The GS energy can be computed exactly in $d=1$ using transfer-matrix methods. ${ }^{9}$ For $H=0$ the free energy of a chain of length $N$ is given by

$$
F_{N}=-\frac{1}{\beta} \ln \left(Z_{N}\right)=-\frac{1}{\beta} \ln \left(Z_{N}^{+}+Z_{N}^{-}\right) \simeq-\frac{1}{2 \beta} \ln \left(Z_{N}^{+} Z_{N}^{-}\right),
$$

where $Z_{N}$ is the partition function with free boundary conditions and $Z_{N}^{ \pm}$are the partition functions with the spin at site $N$ fixed up (down). The last step in Eq. (3) uses the approximation $Z_{N}^{+}+Z_{N}^{-} \simeq \sqrt{Z_{N}^{+} Z_{N}^{-}}$which holds in the large $N$ limit since $Z_{N}^{ \pm}$both diverge with the ratio $Z_{N}^{+} / Z_{N}^{-}$being finite. The two functions $Z_{N}^{+}$and $Z_{N}^{-}$satisfy the following recursive relation:

$$
Z_{N}^{ \pm}=e^{ \pm \beta h_{N}}\left(Z_{N-1}^{+} e^{ \pm \beta J}+Z_{N-1} e^{\mp \beta J}\right) .
$$

From Eq. (4) it follows: 


$$
Z_{N}^{+} Z_{N}=Z_{N-1}^{+} Z_{N-1}\left[2 \cosh (\beta J)+2 \cosh \left(2 \beta x_{N}\right)\right],
$$

where $x_{N}=(1 / 2 \beta) \ln \left(Z_{N}^{+} / Z_{N}^{-}\right)$, which gives for the total free energy,

$$
F_{N}=F_{N-1}-\frac{1}{2 \beta} \ln \left[2 \cosh (\beta J)+2 \cosh \left(2 \beta x_{N}\right)\right],
$$

so that one can define a free energy per site,

$$
f=-\frac{1}{2 \beta} \ln \left[2 \cosh (\beta J)+2 \cosh \left(2 \beta x_{N}\right)\right] .
$$

Here, $x_{N}$ is a stochastic quantity satisfying the equation

$$
x_{N}=h_{N}+g\left(x_{N-1}\right),
$$

where $g(x)=(1 / 2 \beta) \ln \left[\left(e^{2 \beta(x+J)}+1\right) /\left(e^{2 \beta x}+e^{2 \beta J}\right)\right]$. When $R$ $\rightarrow 0$, Eq. (8) has a fixed point solution of $x_{\infty}=g\left(x_{\infty}\right)$. It is easy to check that $x_{\infty}=0$ is the only solution for any $J$ and $\beta$ finite, corresponding to the absence of a phase transition.

When $R$ is nonzero $x_{N}$ is a random variable with an associated distribution $W_{N}(x)$, where

$$
W_{N}(x) d x=\operatorname{Prob}\left(x<x_{N}<x+d x\right) .
$$

$W_{N}(x)$ satisfies the recursive functional equation

$$
W_{N+1}(x)=\int_{-\infty}^{\infty} d h P(h) \int_{-\infty}^{\infty} d x_{1} W_{N}\left(x_{1}\right) \delta\left[x-h-H-g\left(x_{N}\right)\right],
$$

where we have added a uniform field $H$ to the random field so that in the thermodynamic limit, $W_{\infty}$ is given by the fixedpoint equation

$$
W_{\infty}(x)=\int_{-\infty}^{\infty} d x_{N} W_{\infty}\left(x_{1}\right) P\left[x-h-H-g\left(x_{N}\right)\right] .
$$

Once $W_{\infty}$ is known, any thermodynamic quantity can be computed. In particular, the free energy per spin is given by

$$
\langle f\rangle=-\frac{1}{\beta} \int_{-\infty}^{\infty} d x W_{\infty}(x)[\cosh (2 \beta)+\cosh (2 \beta x)] .
$$

The magnetization at a site 0 of an infinite lattice is given by

$$
\left\langle s_{0}\right\rangle=\frac{Z^{+}-Z^{-}}{Z^{+}+Z^{-}}=\frac{\sqrt{Z^{+} / Z^{-}}-\sqrt{Z^{-} / Z^{+}}}{\sqrt{Z^{+} / Z^{-}}+\sqrt{Z^{-} / Z^{+}}}=\tanh \left[\frac{1}{2} \ln \left(Z^{+} / Z^{-}\right)\right],
$$

where $Z^{+-}$are, respectively, the partition functions with the spin at 0 fixed up (down). These are given by

$$
Z^{+-}=e^{ \pm \beta h_{0}}\left(e^{ \pm \beta J} Z_{r}^{+}+e^{\mp \beta J} Z_{r}\right)\left(e^{ \pm \beta J} Z_{l}^{+}+e^{\mp \beta J} Z_{l}\right),
$$

where $Z_{r, l}^{ \pm}$are the partition functions for the semi-infinite right (left) lattice, with the spin at site $1(-1)$ fixed up (down). This gives

$$
\left\langle s_{0}\right\rangle=\tanh \left\{\beta\left[h_{0}+g\left(x_{r}\right)+g\left(x_{l}\right)\right]\right\},
$$

where $x_{r}\left(x_{l}\right)$ refers to the semi-infinite right (left) lattice. Finally, the magnetization for the infinite lattice is obtained averaging over the quenched variables $x_{r, l}$ :

$$
\begin{aligned}
m= & \int_{-\infty}^{\infty} d h P(h) \int_{-\infty}^{\infty} d x_{r} W_{\infty}\left(x_{r}\right) \\
& \times \int_{-\infty}^{\infty} d x_{l} W_{\infty}\left(x_{l}\right) \tanh \left\{\beta\left[h+g\left(x_{r}\right)+g\left(x_{l}\right)\right]\right\} .
\end{aligned}
$$

\section{B. Exact results: Demagnetized state}

In $d=1$ the magnetization and the energy per spin as a function of the external field can be derived explicitly through a probabilistic reasoning. We show how to get these results on the saturation loop, focusing on the lower branch (the results on the upper branch can be obtained by symmetry considerations). Similar but much more involved reasoning can be repeated for any minor loop.

The central quantity to consider, in order to solve for the magnetization as a function of the external field $H$ on the hysteresis loop, is the conditional probability for a spin to be up, conditioned to one of its nearest neighbors being down. To calculate this quantity, one can reason as follows: first fix the spin at site $i-1$ down, and define $p_{m}(H)$ as the probability for a spin to be up, given that exactly $m(m=0,1,2)$ of its neighbors are up,

$$
p_{m}(H)=P\left(h_{i}^{\mathrm{eff}}>0\right)=\int_{(z-2 m) J-H}^{\infty} d h \rho(h),
$$

where $z$ is the coordination number $(z=2$ in $d=1)$. Fix now for a moment the spin at site $i$ down as well and look at the spin at site $i+1$. It will be up with probability $U_{0}$ and down with probability $1-U_{0}$. The spin at site $i$ will flip up with probability $p_{1}$ when the spin at $i+1$ is up and $p_{0}$ when it is down. Ultimately, the spin at $i$ will be up (conditioned to the spin at $i-1$ being down $)$ with probability $U_{0}=U_{0} p_{1}+(1$ $\left.-U_{0}\right) p_{0}$. It follows:

$$
U_{0}=\frac{p_{0}}{1-p_{1}+p_{0}} .
$$

Once $U_{0}$ is known, a similar reasoning leads to the (unconditioned) probability $p(H)$ for a spin to be up. Now let us fix the spin at site $i$ down: the spin at site $i-1$ will be up with probability $U_{0}$ and down with probability $1-U_{0}$. The same holds for the spin at site $i+1$. Thus,

$$
p(H)=U_{0}^{2} p_{2}+2 U_{0}\left(1-U_{0}\right) p_{1}+\left(1-U_{0}\right)^{2} p_{0},
$$

from which the magnetization is obtained as $m(H)=2 p(H)$ -1 .

The energy per spin on the saturation loop is obtained as follows. Due to translational invariance,

$$
E=\frac{\langle\mathcal{H}\rangle}{N}=-J\left\langle s_{i} s_{i+1}\right\rangle-H\left\langle s_{i}\right\rangle-\left\langle h_{i} s_{i}\right\rangle .
$$

To calculate the spin-spin correlation $\left\langle s_{i} s_{i+1}\right\rangle$ we introduce the probabilities $\Phi^{++}, \Phi^{+-}, \Phi^{-+}, \Phi^{--}$for adjacent spins to be, respectively, up-up, up-down, down-up, and down-down. These quantities are not independent, since they have to satisfy the obvious identities: $\Phi^{+-}=\Phi^{-+}, \Phi^{++}+\Phi^{+-}=p(H)$, and 
$\Phi^{--}+\Phi^{+-}=1-p(H)$. Thus it is sufficient to calculate one of them, for example $\Phi^{--}$. This is done by separating the four contributions from the possible boundary conditions determined by the values of the spins at sites $i-1$ and $i+1$. When they are both down, the probability for the couple of spins at sites $i$ and $i+1$ to be both down is $U_{0}^{2}\left[1-p_{1}(H)\right]^{2}$, when one is up and the other is down is $2 U_{0}\left(1-U_{0}\right)\left[1-p_{1}(H)\right][1$ $\left.-p_{0}(H)\right]$, and when both of them are up is $\left(1-U_{0}\right)^{2}[1$ $\left.-p_{0}(H)\right]^{2}$. Adding up the four contributions we get $\Phi^{--}=(1$ $\left.-U_{0}\right)^{2}$. This fixes the other probabilities to be $\Phi^{+-}=\Phi^{-+}$ $=2 p-1+\left(1-U_{0}\right)^{2}$, and $\Phi^{++}=1-p-\left(1-U_{0}\right)^{2}$. Thus, the spinspin correlation is

$$
\left\langle s_{i} s_{i+1}\right\rangle=\Phi^{++}+\Phi^{--}-2 \Phi^{+-}=4\left[p-\left(1-U_{0}\right)^{2}\right]-3 .
$$

The average value $\left\langle h_{i} s_{i}\right\rangle$ can be obtained by averaging over the field $h^{\prime}$ the product of $h^{\prime}$ times the average value of the spin $s_{i}$ over the local fields other than $h_{i}$, once the field at $i$ is fixed at the value $h^{\prime}$ :

$$
\left\langle h_{i} s_{i}\right\rangle=\int_{-\infty}^{+\infty} d h^{\prime} \rho\left(h^{\prime}\right) h^{\prime}\left\langle s_{i} \mid h^{\prime}\right\rangle .
$$

The conditional average $\left\langle s_{i} \mid h^{\prime}\right\rangle$ is given by $2 p\left(H \mid h^{\prime}\right)-1$ where $p\left(H \mid h^{\prime}\right)$ is the conditional probability for a spin to be up at an external field $H$, given that its local random field is fixed at the value $h^{\prime}$. From Eq. (9) this is trivially given by

$$
\begin{aligned}
p\left(H \mid h^{\prime}\right)= & U_{0}^{2} \theta\left(h^{\prime}+H+2 J\right)+2 U_{0}\left(1-U_{0}\right) \theta\left(h^{\prime}+H\right) \\
& +\left(1-U_{0}\right)^{2} \theta\left(h^{\prime}+H-2 J\right),
\end{aligned}
$$

which finally gives

$$
\begin{aligned}
\left\langle h_{i} s_{i}\right\rangle= & 2 U_{0}^{2} \int_{-H-2 J}^{+\infty} d h^{\prime} \rho\left(h^{\prime}\right) h^{\prime}+4 U_{0}\left(1-U_{0}\right) \int_{-H}^{+\infty} d h^{\prime} \rho\left(h^{\prime}\right) h^{\prime} \\
& +2\left(1-U_{0}\right)^{2} \int_{-H+2 J}^{+\infty} d h^{\prime} \rho\left(h^{\prime}\right) h^{\prime}-\bar{h}^{\prime} .
\end{aligned}
$$

In particular, for a Gaussian distribution with $\overline{h^{\prime}}=0$ and variance $R$ the integrals can be performed analytically and the result is

$$
\begin{aligned}
\left\langle h_{i} S_{i}\right\rangle= & \sqrt{\frac{2}{\pi}} \operatorname{Re}^{-H^{2} / 2 R^{2}}\left[2 U_{0}^{2} e^{2 J / R^{2}} \cosh \left(2 J H / R^{2}\right)+e^{2 J\left[J-\left(H / 2 R^{2}\right)\right]}\right. \\
& \left.\times\left(1-2 U_{0}^{2}\right)+2 U_{0}\left(1-U_{0}\right)\right] .
\end{aligned}
$$

The energy per site on the lower branch of the saturation loop is in general given by

$$
\begin{aligned}
E(H)= & -4 J\left[p(H)-\left(1-U_{0}\right)^{2}\right]+3 J-H(2 p(H)-1) \\
& -2 U_{0}^{2} \int_{-H-2 J}^{+\infty} d h^{\prime} \rho\left(h^{\prime}\right) h^{\prime} \\
& -4 U_{0}\left(1-U_{0}\right) \int_{-H}^{+\infty} d h^{\prime} \rho\left(h^{\prime}\right) h^{\prime} \\
& -2\left(1-U_{0}\right)^{2} \int_{-H+2 J}^{+\infty} d h^{\prime} \rho\left(h^{\prime}\right) h^{\prime}+\bar{h}^{\prime} .
\end{aligned}
$$

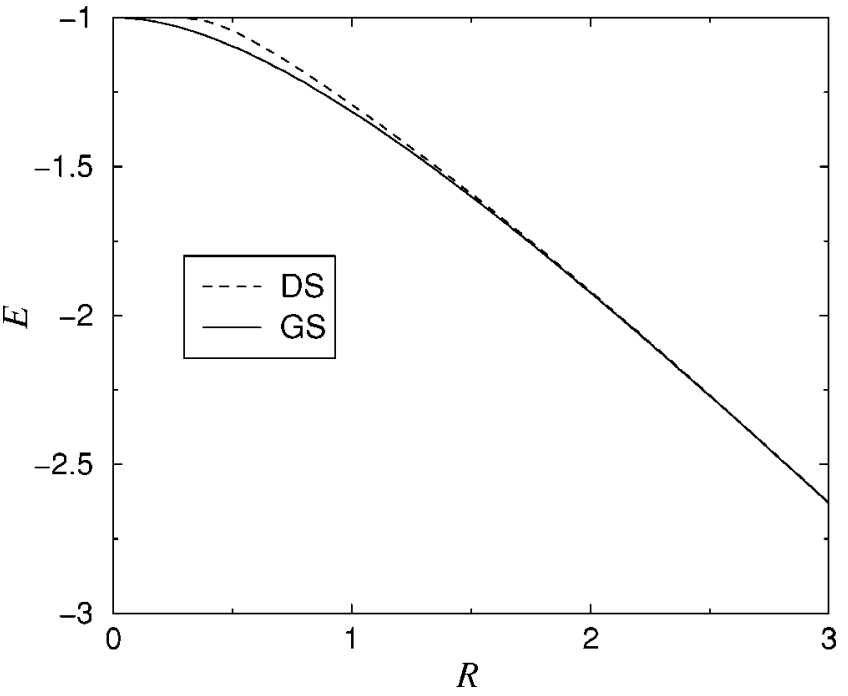

FIG. 2. The energy of the GS is compared with the one of DS. The values are computed exactly in $d=1$ as a function of the disorder width $R$.

Similar but much more involved reasonings can be repeated for any minor loop-eventually for a series of nested loops leading to the demagnetized state-providing a series of recursive equations for the magnetization, the spin-spin and the spin-field correlations, which are the quantities needed to compute the energy. If the external field is changed through a nested succession $H=H_{0} \rightarrow H_{1} \rightarrow H_{2} \rightarrow \cdots H_{n} \cdots$ $\rightarrow 0$, with $H_{2 n}>H_{2 n+2}>0, H_{2 n-1}<H_{2 n+1}<0$, and $d H \equiv H_{2 n}$ $-H_{2 n+2} \rightarrow 0$, the spin-spin correlations are given recursively by

$$
\begin{aligned}
\left\langle s_{i} s_{i+1}\right\rangle_{H_{2 n}}-\left\langle s_{i} s_{i+1}\right\rangle_{H_{2 n-1}}= & 4 U_{2 n}^{2}\left[p_{2}\left(H_{2 n}\right)-p_{2}\left(H_{2 n-1}\right)\right] \\
& -4 D_{2 n-1}^{2}\left[p_{0}\left(H_{2 n}\right)-p_{0}\left(H_{2 n-1}\right)\right],
\end{aligned}
$$

where $U_{k}$ and $D_{k}$ are, respectively, the probabilities for a spin to be up (down) conditioned to one of its neighbors being down, and satisfy in turn a set of recursive equations. Similar equations hold for magnetization and spin-field correlation, leading to a complicated recursive formula for the energy. The results of such calculations are shown in Figs. 2 and 3, where the energy of the demagnetized state is compared with the energy of the ground state evaluated in the preceding section.

\section{Simulations: How optimized is the demagnetized state?}

While the energies of the GS and DS can be obtained exactly in $d=1$, we use numerical simulations to obtain a more detailed picture of the differences between the two states. We consider a system of size $N=10^{4}$ spins and obtain numerically the GS and the DS averaging the results over 1000 realizations of the random fields. In this way, we can recover to a great precision the exact results (see Fig. 3), indicating that the system size and statistical sampling employed are adequate to fully characterize the system. 


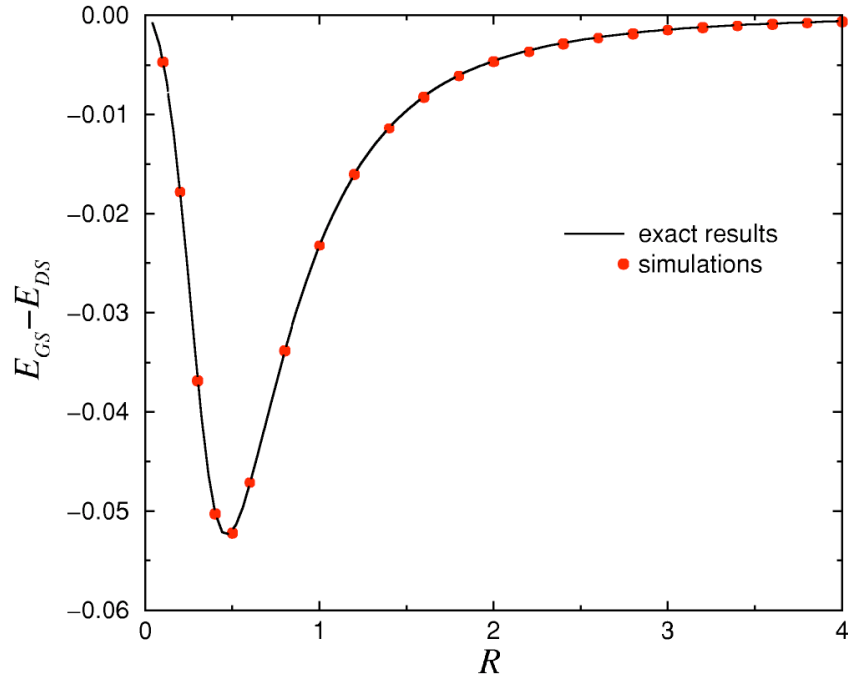

FIG. 3. The energy difference between the GS and the DS computed exactly in $d=1$ is compared with the numerical simulations.

In one dimension the comparison of the domain structure of DS and the GS is the easiest since the domain walls are just pointlike. For the GS we know that it is optimized such that all the large enough local random-field fluctuations nucleate domains of the same sign. The rest of the random landscape is split up into regions that align themselves with such fluctuations depending on the sign of the random-field excess, $\sum_{i \in \text { region }} h_{i}$. As a result the Zeeman energy of domains, defined as $E_{Z}=\sum_{i \in \text { domain }} h_{i}$, is linear in domain size, $E_{Z} \sim l_{d}$, and the asymptotic mean domain length follows the Imry-Ma prediction $\left\langle l_{\mathrm{GS}}\right\rangle \sim 1 / R^{2}$. Moreover, since the random landscape has a finite correlation length, the domain size distribution is exponential. ${ }^{36}$

Any qualitative differences in the DS will follow from three separate mechanisms: (1) shifts of domain walls, (2) creation of domains inside intact GS domains, and (3) destruction of GS domains (Fig. 4). From the point of view of "optimization" the first one is of trivial concern, since it would have little effect, e.g., on the scaling of $E_{Z, D S}$, where the subscript underlines that $E_{Z}$ is computed for the DS. The second one is more detrimental if the energy difference to the GS is considered. In addition to the cost of the two domain walls it subtracts a contribution from the Zeeman energy of the domain that persists and surrounds (in the GS) the one that is not created in the DS. The third one would make the largest change to the total energy, since for $l_{\mathrm{DS}} \gg 1$ the energy

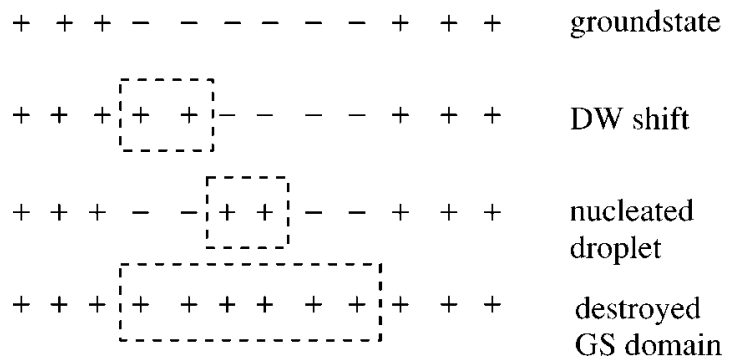

FIG. 4. An illustration of the possible mechanisms for the deviations between GS and DS.

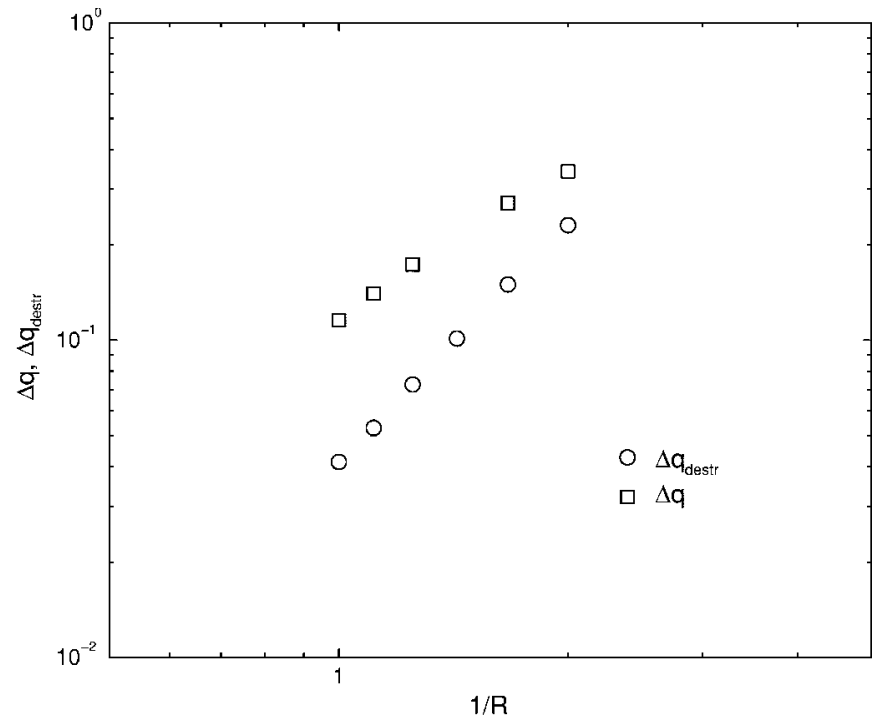

FIG. 5. The average change in the spin-spin overlap between the GS and the DS $(\Delta q)$ and the contribution to that from completely "destroyed" GS domains $\left(\Delta q_{\text {destr }}\right)$, as a function of $R$.

of a domain consists mostly of its Zeeman energy.

Numerical studies of the DS domain structure indicate that with decreasing $R$ the average domain size increases faster than in the GS, while the size distribution $P\left(l_{d}\right)$ remains exponential. This is accompanied by a reduction in the overlap $q=\left(\left\langle s_{\mathrm{GS}} s_{\mathrm{DS}}\right\rangle+1\right) / 2$ between these two states. For $R$ large the overlap is close to unity; strong local fields $h_{i}$ align the spins in the same way regardless of the mechanism by which the spin state is created. For $R$ small the local field is no longer strongly correlated with the orientation of the spin, and thus whether the GS and DS are locally aligned depends on how optimized the latter is.

The fundamental mechanism for the deviations between the states seems to be the "destruction" of GS domains, at least for small $R$ (see Fig. 4 again). This is demonstrated in Fig. 5 by depicting the change $\Delta q$ in the overlap that comes solely from missing GS domains. The conclusion from this dominance is that the demagnetized states typically miss regions in which the integrated field fluctuation is large which, as such, leads in the GS to the formation of GS domain. Therefore the overlap should get smaller the larger the scale length on which one compares the DS and GS, as confirmed by Fig. 6, which shows the overlap between a DS domain and the GS as a function of the length $l_{\mathrm{DS}}$ of the DS domain.

The importance of such destroyed domains can also be seen in the total contribution to the energy difference between the DS and GS. For $R$ small this is again dominated by missing GS domains. In general the difference between the energies of the GS and DS derives from the combination of domain walls and Zeeman energy. Figure 7 shows that for $l_{\mathrm{DS}}$ small the DS domains do not have much Zeeman energy. This changes if $l_{\mathrm{DS}}$ is larger, in which regime the scaling approaches the Imry-Ma -like scaling $\left(l_{\mathrm{DS}}^{0.5}\right)$. The implication is that the field energy of large domains in the DS selfaverages, and comes from a sum of random contributions (i.e., the domains contain regions where the actual randomfield sum is opposite to the spin orientation, such as the 


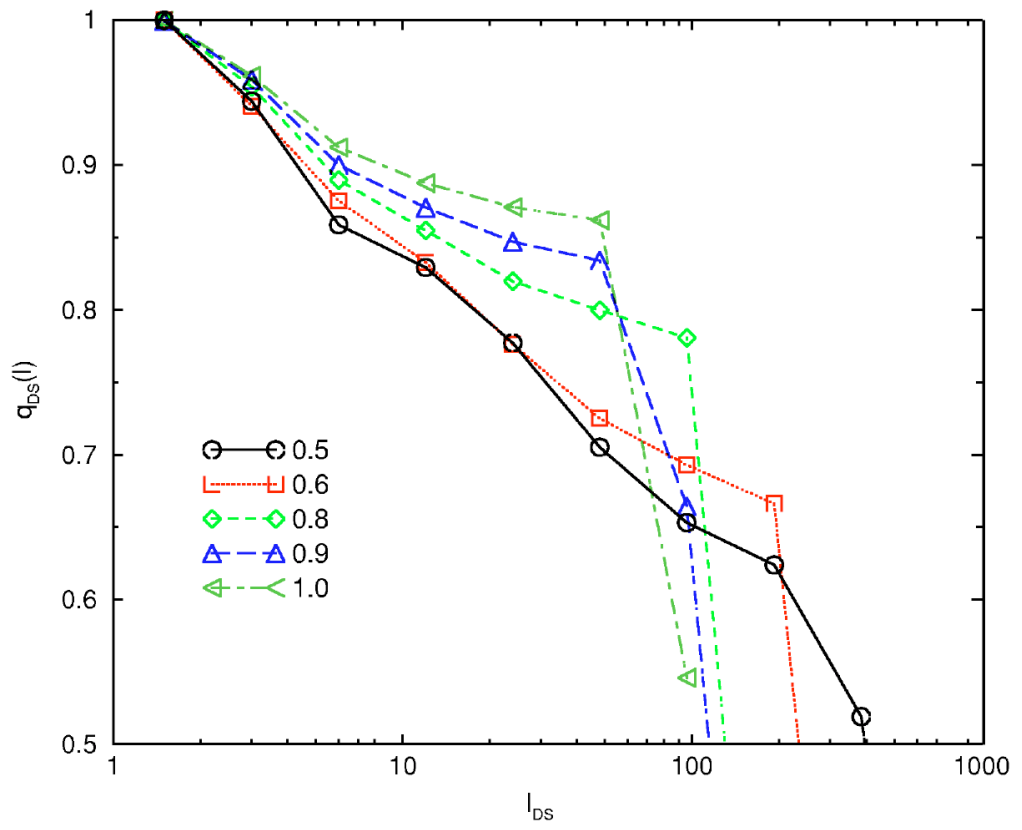

FIG. 6. (Color online) The average overlap of a DS domain of size $l_{\mathrm{DS}}$ with the GS domain spin state at the same locations for $R=0.5,0.6,0.8$, $0.9,1.0$. The overlap decreases with $l_{\mathrm{DS}}$. missing GS domains). The crossover between the small $l_{\mathrm{DS}}$ behavior and the asymptotic scaling is located close to $\left\langle l_{\mathrm{DS}}\right\rangle$.

\section{AROUND THE DISORDER INDUCED TRANSITION}

\section{A. Simulations in $d=3$}

The RFIM displays a disorder induced phase transition both in the GS and in the hysteresis loop, which can also be observed by analyzing the DS. ${ }^{21,22,39}$ If the GS and the DS are always paramagnetic, the transition is absent, as for $d$ $<3$. Thus we perform numerical simulations in $d=3$, with the aim to characterize the difference between DS and GS around the disorder induced transition.

In $d=3$ for low disorder, the GS is ferromagnetic, while for higher disorder it becomes paramagnetic. For Gaussian disorder, the transition point has been located numerically ${ }^{6-8}$ at $R_{c}^{(\mathrm{GS})} \simeq 2.28$ (see below). It is possible to define the usual set of critical exponents characterizing the phase transition and compute the values by exact GS calculations. For instance, the magnetization $M \equiv\langle|m|\rangle$, with $m \equiv \Sigma_{i} s_{i} / N$, scales close to the transition point as

$$
M=A r^{\beta},
$$

where $r \equiv\left(R-R_{c}\right) / R_{c}$ is the reduced order parameter and $A$ is a nonuniversal constant. The correlation length defines another exponent $\xi=(B r)^{-\nu}$, where $B$ is another nonuniversal constant which rules the finite-size scaling of the model, so that

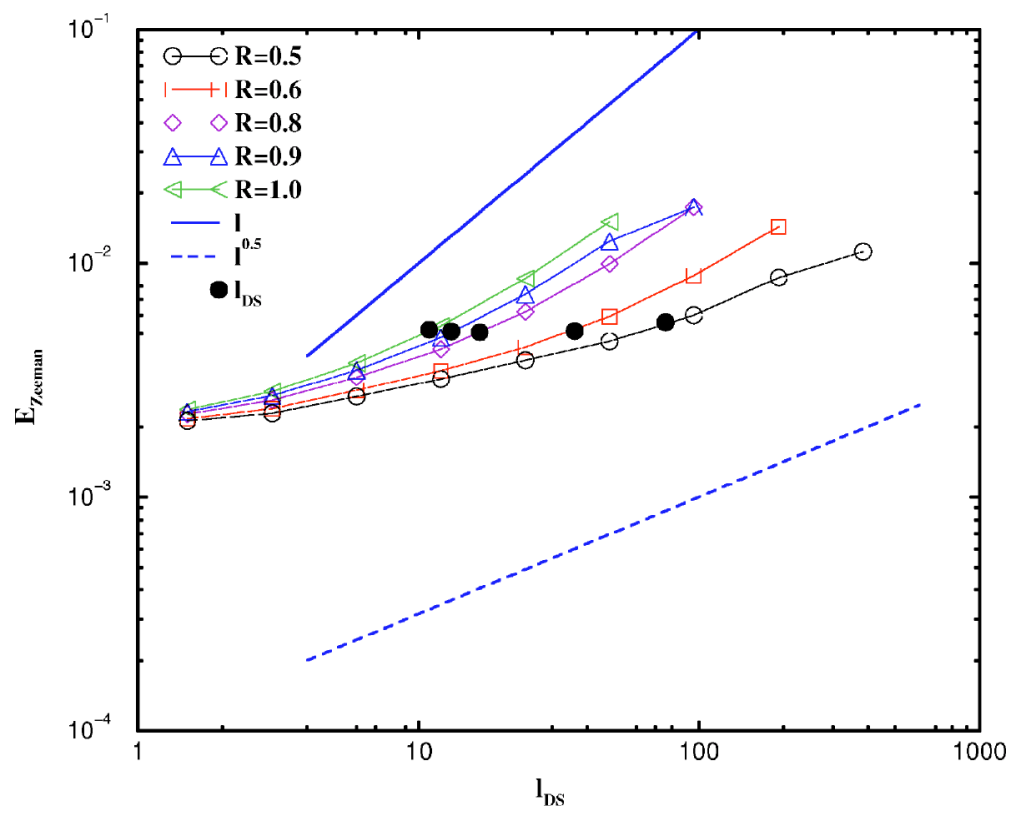

FIG. 7. (Color online) The Zeeman energy of DS domains of the size, $l_{\mathrm{DS}}$. The black circles mark the average DS domain size for a given $R$. The two lines above and below the data indicate optimal, linear (GS-like) scaling and the ImryMa-like $l^{1 / 2}$ scaling, respectively. 


$$
M=A L^{-\beta / \nu} f\left[B L^{1 / \nu}\left(R-R_{c}\right) / R_{c}\right] .
$$

Simulations ${ }^{6-8}$ yield $\nu^{(\mathrm{GS})} \simeq 1.17$ and $\beta^{(\mathrm{GS})}=0.02$.

A disorder induced transition is also found in the hysteresis loop. At low disorder the loop shows a macroscopic jump, which disappears at a critical value of the disorder. This transition reflects itself on the DS, which is ferromagnetic when the main loop has a jump and is paramagnetic otherwise. The transition point has been obtained numerically in $d=3$ as $R_{c}^{(\mathrm{DS})} \simeq 2.16$ and the critical exponents have been measured. In particular, Ref. 39 reports data collapses with $\beta_{(\mathrm{DS})}=0.04$ and $\nu_{(\mathrm{DS})}=1.41$. While there is strong evidence that the exponents measured in the DS should be equal to those measured on the main loop, the relation with the equilibrium transition is not clear.

We notice first that numerical simulations reported in the literature indicate that the transition appears at slightly different locations in the GS and in the DS. Hartmann and Nowak $^{6}$ report $R_{c}^{(\mathrm{GS})}=2.29 \pm 0.04$ for the GS with system size up to $L=80$, Hartmann and Young ${ }^{7}$ refine this value to $R_{c}^{(\mathrm{GS})}=2.28 \pm 0.01$ with sizes up to $L=96$, which is also confirmed by Middleton and Fisher $^{8}$ who estimate $R_{c}^{(\mathrm{GS})}$ $=2.27 \pm 0.04$. For the hysteresis loop the best estimate is $R_{c}$ $=2.16 \pm 0.03$, with system sizes up to $L=320$ and a similar value for the DS. ${ }^{21,39}$ Thus, unless strong finite-size effects take place, one is tempted to conclude that the two transitions take place at two different values of $R_{c}$.

Here we analyze the problem again by numerical simulations, computing the GS and the DS numerically, using the same disorder realizations for the two cases. Simulations are performed for cubic lattices of linear sizes $L=10,20,40,60$, 80 and the results are averaged over several realizations of the random fields. The GS is found exactly using a min-cut/ max-flow algorithm, while demagnetization is performed approximately with the algorithm discussed in Ref. 21 with $d H=10^{-3}$ (see Sec. II). In both cases, we compute the average magnetization as a function of the disorder width (see Fig. 8). In Fig. 9 we collapse the two sets of data into a single curve, using two different values for $R_{c}$ (i.e., $R_{c}^{(\mathrm{GS})}$ $=2.28$ and $\left.R_{c}^{(\mathrm{DS})}=2.16\right)$ but the same values for the exponents (i.e., $\nu=1.37$ and $\beta=0.03$ ). The best value for the ratio of the nonuniversal constant is found to be $A_{\mathrm{DS}} / A_{\mathrm{GS}} \simeq 1$ and $B_{\mathrm{DS}} / B_{\mathrm{GS}}=0.68 \pm 0.02$. The fact that the scaling function is the same for the two cases is a strong indication for universality, going beyond the simple numerical similarity of the exponents. There is always the possibility that in the thermodynamic limit $R_{c}^{(\mathrm{GS})}=R_{c}^{(\mathrm{DS})}$. At the present stage this hypothesis is not supported by the data, since we were not able to collapse all the data into a single curve using the same $R_{c}$.

Next, we compare the statistical properties of the GS and the DS around the transitions. In Fig. 10, we report the value of the overlap as a function of $R$ for different system sizes. When the disorder is decreased from the paramagnetic region, the overlap decreases as for $d=1$. However, for low disorder, the overlap rapidly increases and reaches 1 in the ferromagnetic state. The minimum of the overlap is located in the parameter region corresponding to the transitions (i.e., $R \sim 2.2-2.3$ ). A decrease in the overlap around the transition

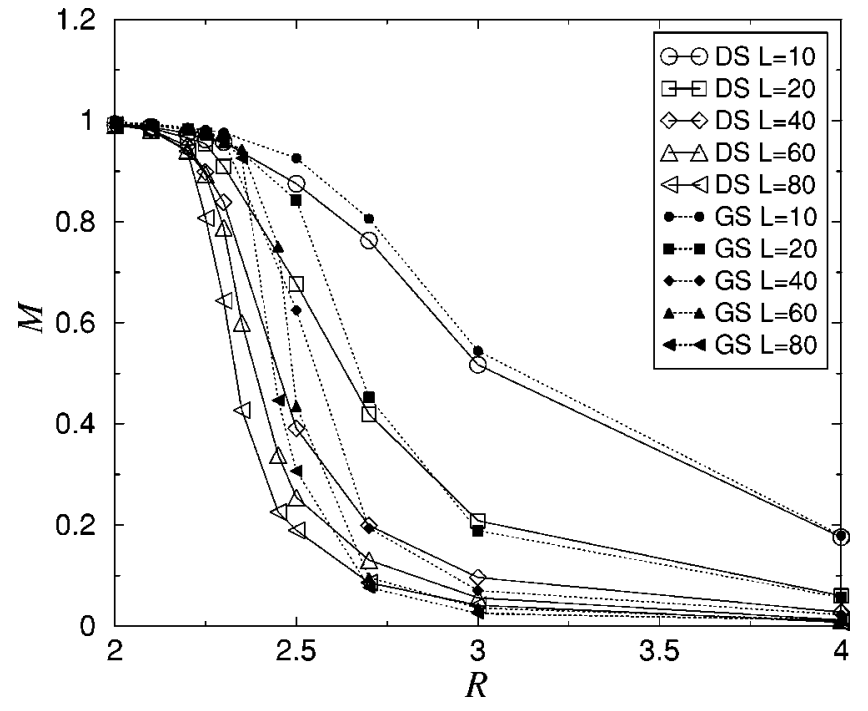

FIG. 8. The magnetization of the GS and the DS in $d=3$ for different system size $L$ and disorder $R$.

can be expected, since for $R_{c}^{(\mathrm{DS})}<R<R_{c}^{(\mathrm{GS})}$ the GS is ferromagnetic $(M>0)$ and the DS is paramagnetic $(M=0)$ as it is also apparent plotting the difference in the magnetization (see Fig. 11).

In summary, three-dimensional simulations indicate that the transitions in the GS and DS are universal, but the critical parameter seems to differ. Consequently the GS and DS differ mostly around the transition, while the difference is smaller in the paramagnetic and ferromagnetic phases.

\section{B. The Bethe lattice}

The RFIM can be solved exactly in the Bethe lattice, displaying a disorder induced transition in the GS and in the

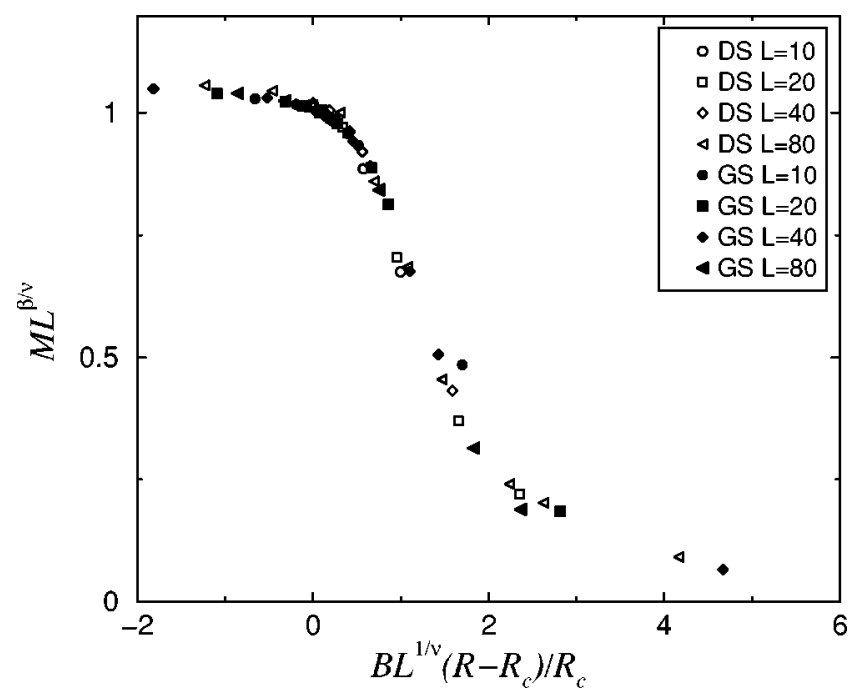

FIG. 9. Numerical results in $d=3$ : The magnetization can be collapsed using $R_{c}=2.28$ (GS) and $R_{c}=2.16$ (DS), $\nu=1.37$, and $\beta$ $=0.03$. The scaling curve is the same for DS and GS indicating universal behavior. The values for the ratios of the nonuniversal constants are $A_{\mathrm{DS}} / A_{\mathrm{GS}}=1$ and $B_{\mathrm{DS}} / B_{\mathrm{GS}}=0.68$. 


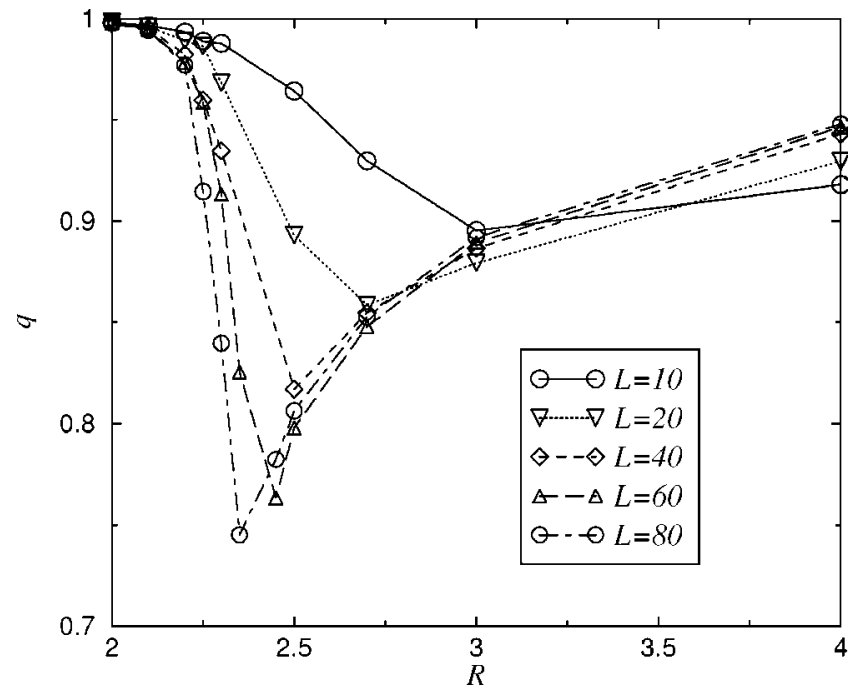

FIG. 10. The overlap between the GS and the DS in $d=3$ for different system sizes.

$\mathrm{DS}^{22}$ It is thus an interesting case to compare the two states around the respective transition directly in the thermodynamic limit. We consider here a Bethe lattice with coordination $z$ and obtain the GS generalizing the $d=1$ case as in Ref. 9. In this case $N$ refers to the generation of the lattice, and $Z_{n}^{ \pm}(i)$ are the partition functions of a branch of generation $n$ with a fixed up (down) spin at a central site $i$. The recursion relation for the $Z_{n}^{ \pm}(i)$ is

$$
Z_{n}^{ \pm}(i)=e^{ \pm \beta h_{i}} \prod_{j \in I(i)}\left[Z_{n-1}^{+}(j) e^{ \pm \beta J}+Z_{n-1}^{-}(j) e^{\mp \beta J}\right]
$$

where for any given site $i$ the sum over $j$ runs over the set $I(i)$ of the $z-1$ nearest neighbors of $i$ away from the center of the lattice. Then, following the $d=1$ case, one can write

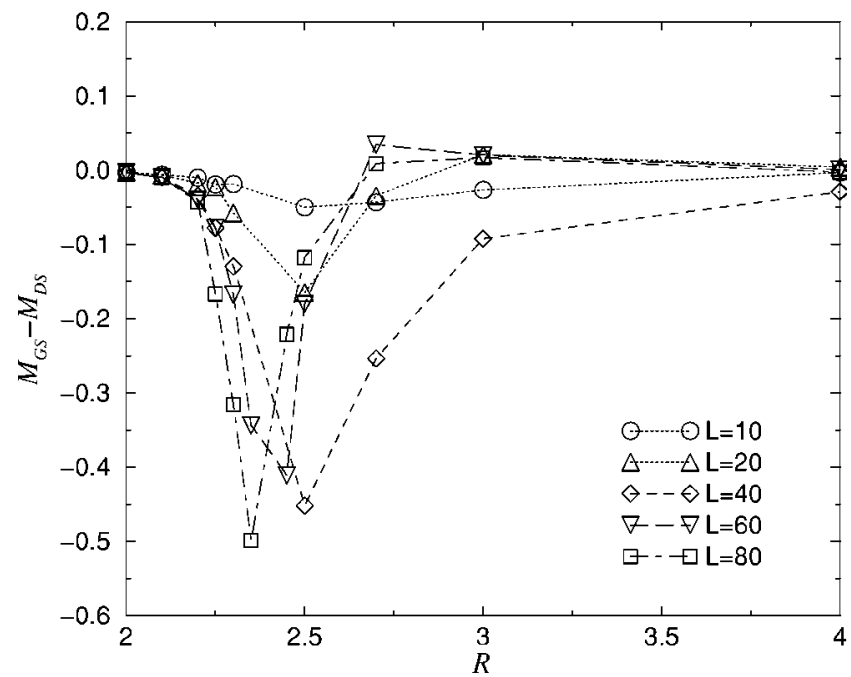

FIG. 11. The difference in magnetization between the GS and the DS in $d=3$ for different system sizes.

$$
F_{n}(i)=\sum_{j \in I(i)}\left[F_{n-1}(j)-\frac{1}{2 \beta} \ln 2\left\{\cosh (\beta J)+\cosh \left[2 \beta x_{n}(j)\right]\right\}\right] \text {, }
$$

where

$$
x_{n}(i)=\frac{1}{2 \beta} \ln \left[Z_{n}^{+}(i) / Z_{n}^{-}(i)\right] \text {, }
$$

so that the contribution at the free energy from site $i$ is

$$
f(i)=-\frac{1}{2 \beta} \ln \left[2 \cosh (\beta J)+2 \cosh \left(2 \beta x_{n}(i)\right)\right] .
$$

$x_{n}(i)$ is a stochastic quantity satisfying the equation

$$
x_{n}(i)=h_{i}+\sum_{j \in I(i)} g\left[x_{n-1}(j)\right]
$$

When $R \rightarrow 0$, Eq. (34) has a fixed point solution of $x_{\infty}=(z$ $-1) g\left(x_{\infty}\right) . x_{\infty}=0$ is a solution for any $J$ and $\beta$. For $\beta<\beta_{c}$ $=(1 / 2) \ln [z /(z-2)]$, there are also two stable solutions $\pm x_{\infty}$ $\neq 0$ corresponding to the appearance of a ferromagnetic phase.

To perform quenched averages one has to solve for the probability distribution of $W_{n}\left(x_{n}\right)$, where $W_{n}(x) d x=\operatorname{Prob}(x$ $\left.<x_{n}<x+d x\right)$, which satisfies the recursive functional equation

$$
\begin{aligned}
W_{n+1}(x)= & \int_{-\infty}^{\infty} d h P(h) \int_{-\infty}^{\infty} d x_{1} W_{n}\left(x_{1}\right) \cdots \int_{-\infty}^{\infty} d x_{z-1} W_{n}\left(x_{z-1}\right) \\
& \times \delta\left[x-h-H-\sum_{k=1}^{z-1} g\left(x_{k}\right)\right]
\end{aligned}
$$

so that in the thermodynamic limit $W_{\infty}$ is given by the fixedpoint equation

$$
\begin{aligned}
W_{\infty}(x)= & \int_{-\infty}^{\infty} d x_{1} W_{\infty}\left(x_{1}\right) \cdots \int_{-\infty}^{\infty} d x_{z-1} W_{\infty}\left(x_{z-1}\right) \\
& \times P\left[x-h-H-\sum_{k=1}^{z-1} g\left(x_{k}\right)\right] .
\end{aligned}
$$

Once $W_{\infty}$ is known, any thermodynamic quantity can be computed. In particular, the free energy per spin is given again by Eq. (12) and the magnetization at the central site of an infinite lattice is given by Eq. (13), where $Z^{\uparrow \downarrow}$ are the partition function with the spin at 0 fixed up (down), respectively. They are given by

$$
Z^{\uparrow \downarrow}=e^{ \pm \beta h_{0}} \prod_{k=1, z}\left(e^{ \pm \beta J} Z_{k}^{+}+e^{\mp \beta J} Z_{k}\right)
$$

and $Z_{k}^{ \pm}$for $k=1, \ldots, z$ are the partition functions of the $z$ branches attached to the central site 0 , with the boundary spin fixed up (down). This gives for the magnetization at the central site $\left\langle s_{0}\right\rangle$, 


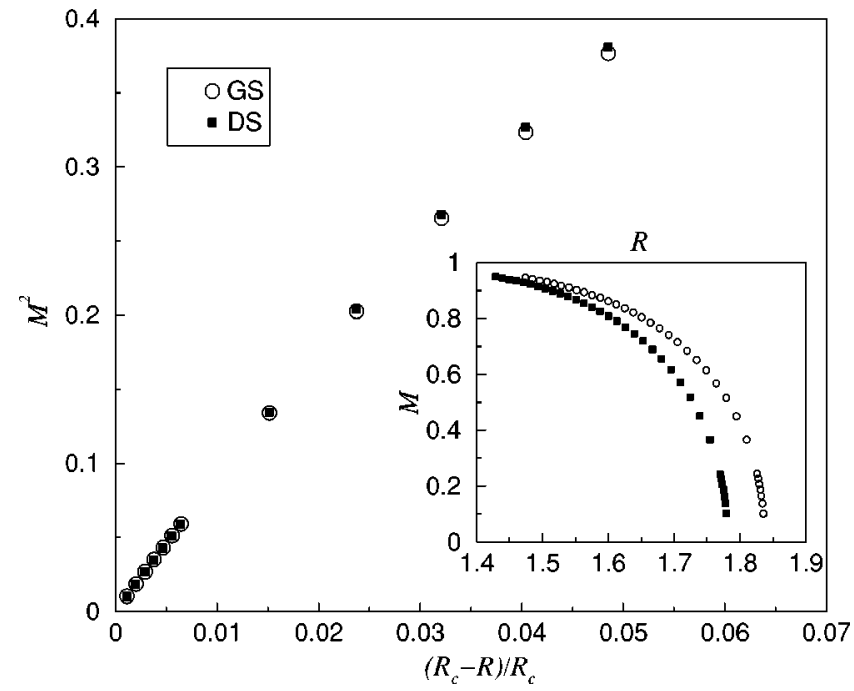

FIG. 12. The magnetization of the GS and the DS computed exactly on the Bethe lattice with $z=4$ in the thermodynamic limit, showing the ordering of the critical point (see the inset). When the data are plotted against the reduced parameter $\left(R_{c}-R\right) / R_{c}$ the curves superimpose. The result implies that for the Bethe lattice $A_{\mathrm{GS}}=A_{\mathrm{DS}}$.

$$
\left\langle s_{0}\right\rangle=\tanh \left\{\beta\left[h_{0}+\sum_{k=1, z} g\left(x_{k}\right)\right]\right\} .
$$

The magnetization for the infinite lattice can then be obtained averaging over the quenched variables $x_{r, l}$,

$$
\begin{aligned}
M= & \int_{-\infty}^{\infty} d h P(h) \int_{-\infty}^{\infty} d x_{1} W_{N}\left(x_{1}\right) \cdots \int_{-\infty}^{\infty} d x_{z} W_{N}\left(x_{z}\right) \\
& \times \tanh \left\{\beta\left[h+\sum_{k=1, z} g\left(x_{k}\right)\right]\right\} .
\end{aligned}
$$

For a Gaussian random-field distribution the fixed-point equation cannot be solved explicitly and we thus resort to a numerical integration. We obtain $W_{\infty}(x)$ for $z=4$, and for different values of $R$, and compute the magnetization using Eq. (39). In Fig. 12 we compare the magnetization of the GS with the one of the remnant magnetization in the DS, computed in Ref. 22. As observed in the simulations in $d=3$, the transition occurs at two different locations (see the inset of Fig. 12), for $z=4, R_{c}^{(\mathrm{DS})}=1.781258 \ldots{ }^{22}$, and $R_{c}^{(\mathrm{GS})} \simeq 1.8375$, with the mean-field exponent $(\beta=1 / 2)$. When plotted against $\left(R-R_{c}\right) / R_{c}$ the two curves superimpose close to the critical point. This indicates that, though not required by universality, in the Bethe lattice $A_{\mathrm{GS}}=A_{\mathrm{DS}}$, as also found in $d=3$.

To investigate possible finite-size scaling we have performed numerical simulations in the Bethe lattice, following the method of Ref. 25. Collapsing the order-parameter curve as in $d=3$, using a scaling form similar to Eq. (29), does not appear to be possible in the Bethe lattice, because the scaling region is very narrow. Thus to test finite-size scaling, we have computed the distribution of the magnetization $m$ at the respective critical points, $R_{c}^{(\mathrm{DS})}$ and $R_{c}^{(\mathrm{GS})}$ for different lattice sizes $N$. The distributions can all be collapsed into the same curve (see Fig. 13), using the form $P(|m|)=f(|m| / M) / M$.

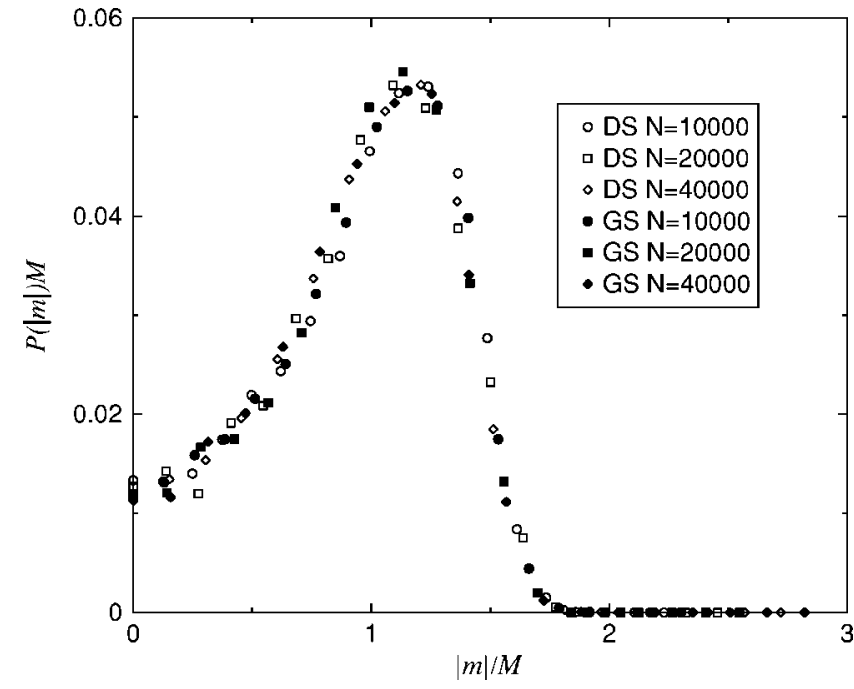

FIG. 13. The distributions of the magnetization in the DS and the GS at their respective critical points on the Bethe lattice, obtained numerically for different lattice sizes $N$, can be all collapsed together.

\section{REACHING THE GROUND STATE BY NONEQUILIBRIUM DYNAMICS}

We have shown that the demagnetization procedure, as a peculiar type of field history, does not necessarily bring the system to the GS, because the DS and GS in general correspond to different microscopic configurations. Now the obvious question is: can we reach the GS by nonequilibrium dynamics? Or, in other words, is there any field history, starting from saturation, which can bring the system to the GS? The answer to this question requires to clarify the relation between the spin configurations visited along the nonequilibrium dynamics driven by the field, and the locally stable states, given as solutions of Eq. (2). In fact, only a limited fraction of stable configurations may be reached by a field history. The problem has been analyzed in detail using an algorithm, called the reverse field history (RFH), which is able to calculate the simplest sequence of reversal fields bringing to a generic stable state, if it exists. ${ }^{40,41} \mathrm{We}$ apply this algorithm to the case of GS, calculated independently by exact combinatorial algorithms.

\section{A. RFH Algorithm}

Consider the spin configuration $\mathbf{s}$ (the set of $N$ Ising spins) reached after the application of a field history consisting in a sequence of reversal fields $\{H\}=\left\{H_{1}, \ldots, H_{n}\right\}$, starting from the saturation, and with $H_{n}=0$. Let us define the generating function $\mathbf{s}=f(\{H\})$, and the stable states it generates as $H$ states. Due to adiabatic dynamical response and return point memory, this state $\mathbf{s}$ contains the memory of a subset of the reversal fields. In fact not all the reversal fields determine the final state s. For example, the reversal fields which give rise to closed minor loops do not influence the final state, because their memory is erased. Thus only the memory of the set of reversal fields $\left\{H_{S}\right\}$ which are not erased is contained in the final state. The inverse function $\left\{H_{S}\right\}=g(\mathbf{s})$ allows to obtain, 


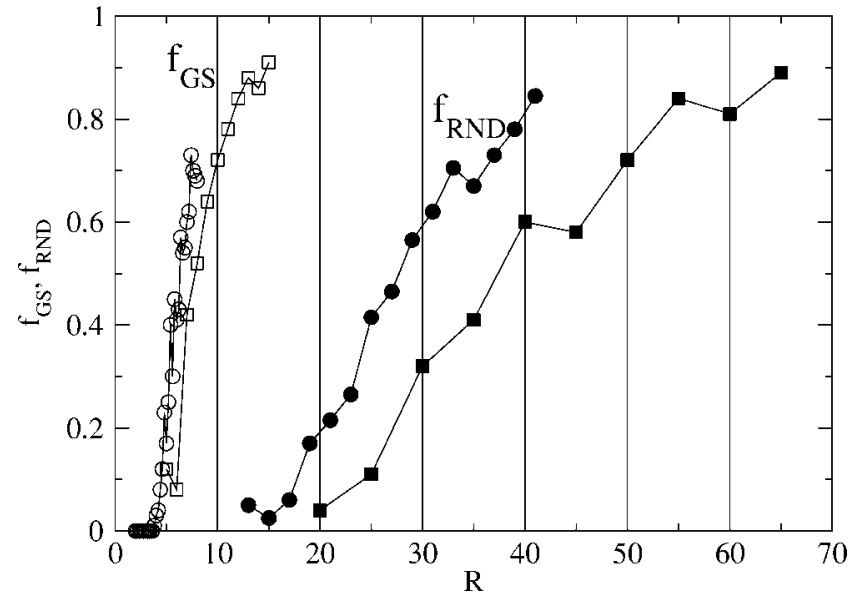

FIG. 14. Fraction of reachable states, averages over 100 realizations of disorder. Full symbols: fraction $f_{\mathrm{RND}}$ of reachable locally stable states, generated by random sampling the set of local minima. Open symbols: fraction $f_{\mathrm{GS}}$ of ground states reachable considering different realizations. System sizes are $N=10000$ (squares) and $N$ $=5000$ (circles).

starting from a spin configuration s belonging to the $H$ states ensemble, the set of reversal fields $\left\{H_{S}\right\}$ which have been actually stored in the state and that-if applied as a field history-will reproduce the original state, i.e., $\mathbf{s}=f(g(\mathbf{s}))$. We define this set of reversal fields $\left\{H_{S}\right\}$ as the minimal field history.

The RFH algorithm takes as input a configuration $\mathbf{s}$ at $H$ $=0$ and gives as output - when it exists - the reversal field history from saturation to the state $\mathbf{s}$. The formulation of the algorithm is based on the order-preserving character of the dynamics, ${ }^{13}$ and is therefore, applicable to a wide range of models beyond the RFIM. An interesting result of the RFH algorithm is obtained when it is applied to a state $\mathbf{s}$ not belonging to the $H$ states (i.e., where no field history exists). The iterated search for the reversal field sequence enters an infinite loop and, in this case, it can be shown that no field history leading to the state exists. ${ }^{40}$

\section{B. Simulation results in one dimension}

We applied the RFH algorithm to the GS in one dimension, with $N=5000,10000$ spins, averaging the results for 100 different realizations of the same disorder $R$. The GS was obtained by the max-flow min-cut procedure for each realization, as described. At each disorder value $R$, we compute the fraction $f_{\mathrm{GS}}$ of the realizations in which the GS is reachable. For comparison, we also consider the fraction $f_{\mathrm{RND}}$ of the reachable locally stable states, generated by random sampling the set of local minima. The results are shown in Fig. 14.

Remarkably, the GS does not result to be systematically field reachable, and the fraction $f_{\mathrm{GS}}$ depends on the disorder (see Fig. 14 for two system sizes). The curve's shape reasonably has limits equal to $f_{\mathrm{GS}}=0$ for $R \rightarrow 0$, where demagnetization becomes impossible, and $f_{\mathrm{GS}}=1$ for $R \rightarrow \infty$, where hysteresis disappears and each state is field reachable. One may argue that the fact that the GS is sometimes reachable is a pure effect of the finite system size. To this end, let us first consider the dependence of $f_{\mathrm{RND}}$ on $R$. For the random states the fraction $f_{\mathrm{RND}}$ sensibly changes with $R$, but following a different curve with respect to $f_{\mathrm{GS}}$ (Fig. 14). If there were no correlation between GS and the $H$ states the two curves would be coincident. The dependence of $f_{\text {RND }}$ on $R$ reflects the fact that the number of $H$ states depends on the disorder value and on the system size, ${ }^{42}$, and only at large disorder, where the number of locally stable states decreases, the ratio between $H$ states and stable states is significantly greater than zero. We cannot make a similar estimation for the fraction $f_{\mathrm{GS}}$, but we note that its shape is not very different from $f_{\mathrm{RND}}$, even if it has different offset in $R$. This point reflects the fact that the GS has a probability to be field reachable significantly higher than any random stable state for the same $N$ and $R$ values.

\section{CONCLUSIONS}

For disordered systems such as the random-field Ising model one would be interested in both universality in statistical properties and in the question how to "optimize" in the case of a sample with a given distribution of the impurities. In this paper we have studied this problem in detail, by comparing the demagnetized and ground states. Our main findings are the following: First, the GS is globally optimized, and the demagnetization procedure does not perform well unless the optimization problem is rather trivial. This is slightly surprising since the conclusion holds in particular if the GS is paramagnetic. In fact, the DS algorithm does not manage to find the right spin configuration, thus many of the domains of the GS do not appear in the DS, as clearly seen in the $d=1$ case.

Second, in $d=3$ (and with the aid of the Bethe lattice solution), we have demonstrated that the existence of a phase transition for both the DS and GS makes the "phase diagram" of optimization to show a regime where the outcome is less optimal: in the paramagnetic phase of the DS, where the GS is already ferromagnetic since the critical thresholds are ordered such that $R_{c}^{(\mathrm{GS})}>R_{c}^{(\mathrm{DS})}$. In this regime DS and GS are expected to differ strongly in the thermodynamic limit. We also provide numerical evidence that the $d=3$ transition appears to have the same critical exponents in both the GS and DS. ${ }^{43}$ This can be considered both surprising - there being no exact field theoretical way of treating the $d=3$ phase transition-and expected, since the functional renormalization calculations in spite of their shortcomings indicate that the actions are the same. ${ }^{14}$ It seems intriguing that such universality is met exactly in the limit where the optimized character of the DS changes.

The results indicate that for the particular system at hand, where the disorder couples directly to the expected magnetization, "local" optimization methods have difficulties. Of course, as in "hysteretic optimization," one can perturb or "shake" the state obtained from the DS procedure to try to still lower the energy. These attempts are of course usually just heuristic. In the case of the RFIM, the joint approach of optimizing by the DS and computing the GS exactly allows to understand better similarities and differences between 
equilibrium and low-energy nonequilibrium states.

In addition to the ferromagnetic RFIM model, one can consider other systems where two disorder induced phase transitions exist. Numerical simulations and analytical results have shown that a disorder induced transition in the hysteresis loop can be observed in the random bond Ising model, ${ }^{44}$ in the random-field $O(N)$ model, ${ }^{45}$ in the random anisotropy model, ${ }^{46,47}$ and in the random Blume-Emery-Griffith model. ${ }^{44}$ All these systems display as well a transition in equilibrium and it would be interesting to compare their DS and GS.
Interfaces in quenched disorder would provide another interesting example, since the roughness exponent typically differs in and out of equilibrium (i.e., at the depinning threshold). ${ }^{4}$ It would be interesting to measure the roughness of an interface after a demagnetization cycle (i.e., after the field driving the interface is cycled with decreasing amplitude), and compare its properties with those of the groundstate interface. Finally, there is the issue of energetics of excitations in the respective ensembles: the universality of exponents and scaling functions would seem to imply that these also scale similarly.
${ }^{1}$ M. Alava, P. Duxbury, C. Moukarzel, and H. Rieger, in Phase Transitions and Critical Phenomena, edited by C. Domb and J. Lebowitz (Academic, San Diego, 2001), Vol. 18.

${ }^{2}$ G. Bertotti, Hysteresis in Magnetism (Academic, San Diego, 1998).

${ }^{3}$ G. Zarand, F. Pazmandi, K. F. Pal, and G. T. Zimanyi, Phys. Rev. Lett. 89, 150201 (2002).

${ }^{4}$ T. Nattermann, in Spin Glasses and Random Fields, edited by A. P. Young (World Scientific, Singapore, 1997).

${ }^{5}$ A. T. Ogielski, Phys. Rev. Lett. 57, 1251 (1986).

${ }^{6}$ A. K. Hartmann and U. Nowak, Eur. Phys. J. B 7, 105 (1999).

${ }^{7}$ A. K. Hartmann and A. P. Young, Phys. Rev. B 64, 214419 (2001).

${ }^{8}$ A. A. Middleton and D. S. Fisher, Phys. Rev. B 65, 134411 (2002).

${ }^{9}$ R. Bruinsma, Phys. Rev. B 30, 289 (1984).

${ }^{10}$ M. R. Swift, A. Maritan, M. Cieplak, and J. R. Banavar, J. Phys. A 27, 1525 (1994).

${ }^{11}$ Z. Slanic, D. P. Belanger, and J. A. Fernandez-Baca, Phys. Rev. Lett. 82, 426 (1999).

${ }^{12}$ F. Ye et al., Phys. Rev. Lett. 89, 157202 (2002).

${ }^{13}$ J. P. Sethna, K. Damen, S. Kartha, J. A. Krumhansl, B. W. Roberts, and J. D. Shore, Phys. Rev. Lett. 70, 3347 (1993).

${ }^{14}$ K. Dahmen and J. P. Sethna, Phys. Rev. B 53, 14872 (1996).

${ }^{15}$ O. Perkovic, K. A. Dahmen, and J. P. Sethna, Phys. Rev. B 59, 6106 (1999).

${ }^{16}$ J. P. Sethna, K. A. Dahmen, and C. R. Myers, Nature (London) 410, 242 (2001).

${ }^{17}$ A. Berger, A. Inomata, J. S. Jiang, J. E. Pearson, and S. D. Bader, Phys. Rev. Lett. 85, 4176 (2000).

${ }^{18}$ J. Marcos, E. Vives, L. Manosa, M. Acet, E. Duman, M. Morin, V. Novak, and A. Planes, Phys. Rev. B 67, 224406 (2003).

${ }^{19}$ F. J. Pérez-Reche and E. Vives, Phys. Rev. B 67, 134421 (2003).

${ }^{20}$ F. J. Pérez-Reche and E. Vives, cond-mat/0403754 (unpublished).

${ }^{21}$ L. Dante, G. Durin, A. Magni, and S. Zapperi, Phys. Rev. B 65, 144441 (2002).

${ }^{22}$ F. Colaiori, A. Gabrielli, and S. Zapperi, Phys. Rev. B 65, 224404
(2002).

${ }^{23}$ P. Shukla, Physica A 233, 235 (1996).

${ }^{24}$ P. Shukla, Phys. Rev. E 62, 4725 (2000).

${ }^{25}$ D. Dhar, P. Shukla, and J. P. Sethna, J. Phys. A 30, 5259 (1997).

${ }^{26}$ S. Sabhapandit, P. Shukla, and D. Dhar, J. Stat. Phys. 98, 103 (2000).

${ }^{27}$ P. Shukla, Phys. Rev. E 63, 027102 (2001).

${ }^{28}$ M. R. Swift, A. J. Bray, A. Maritan, M. Cieplak, and J. R. Banavar, Europhys. Lett. 38, 273 (1997).

${ }^{29}$ A. A. Middleton, cond-mat/0208182 (unpublished).

${ }^{30}$ A. Maritan, M. Cieplak, M. R. Swift, and J. R. Banavar, Phys. Rev. Lett. 72, 946 (1994).

${ }^{31}$ J. P. Sethna, K. Damen, S. Kartha, J. A. Krumhansl, B. W. Roberts, and J. D. Shore, Phys. Rev. Lett. 72, 947 (1994).

${ }^{32}$ J.-C. Anglś d'Auriac and N. Sourlas, Europhys. Lett. 39, 473 (1997).

${ }^{33}$ P. M. Duxbury and J. M. Meinke, Phys. Rev. E 64, 036112 (2001).

${ }^{34}$ R. Dobrin, J. M. Meinke, and P. M. Duxbury, J. Phys. A 35, L247 (2002).

${ }^{35}$ F. Colaiori, M. J. Alava, G. Durin, A. Magni, and S. Zapperi, Phys. Rev. Lett. 92, 257203 (2004).

${ }^{36}$ G. Schröder, T. Knetter, M. J. Alava, and H. Rieger, Eur. Phys. J. B 24, 101 (2001).

${ }^{37}$ E. Seppälä, Ph.D. thesis, HUT, Helsinki, 2001.

${ }^{38}$ G. Bertotti and M. Pasquale, J. Appl. Phys. 67, 5255 (1990).

${ }^{39}$ J. H. Carpenter and K. A. Dahmen, Phys. Rev. B 67, 020412 (2003).

${ }^{40}$ V. Basso and A. Magni, Physica B 343, 275 (2004).

${ }^{41}$ A. Magni and V. Basso, J. Magn. Magn. Mater. (to be published).

${ }^{42}$ V. Basso and A. Magni (unpublished).

${ }^{43}$ A similar suggestion was made independently in Ref. 20.

${ }^{44}$ E. Vives and A. Planes, Phys. Rev. B 50, 3839 (1994).

${ }^{45}$ R. da Silveira and M. Kardar, Phys. Rev. E 59, 1355 (1999).

${ }^{46}$ E. Vives and A. Planes, Phys. Rev. B 63, 134431 (2001).

${ }^{47}$ R. da Silveira and S. Zapperi, Phys. Rev. B 69, 212404 (2004). 